\title{
Introduction: The Philosophy of Information
}

\author{
Bert Baumgaertner ${ }^{1} \cdot$ Luciano Floridi $^{2}$
}

Published online: 3 March 2016

(C) Springer Science+Business Media Dordrecht 2016

The information revolution has changed the world profoundly and irreversibly at a breath-taking pace. Given its unprecedented scope, vital issues have emerged concerning the creation, management, and utilisation of information. The information revolution has brought enormous benefits and opportunities. However, it has outpaced our understanding of its foundations and consequences. It has raised conceptual issues that are rapidly expanding, evolving, and becoming increasingly serious. Today, philosophy faces the challenge of providing a foundational treatment of the concepts and phenomena underlying the information revolution, in order to foster our understanding and guide the responsible construction of our information society. Philosophy of information meets this challenge. It is a thriving new area of research that investigates the conceptual nature and basic principles of information, including its ethical consequences.

Philosophy of information deals with a broad domain of topics and its diversity is reflected in this issue. It contains a selection of papers presented at the $5^{\text {th }}$ Workshop on the Philosophy of Information, organised by the UNESCO Chair in Information and Computer Ethics, in collaboration with the AHRC project 'Understanding Information Quality Standards and their Challenges' (2011-2013) and Google UK. The papers handle various topics, ranging from a

\section{Bert Baumgaertner}

bbaum@uidaho.edu

Luciano Floridi

luciano.floridi@oii.ox.ac.uk

1 University of Idaho, 875 Perimeter Drive, Moscow, ID 83844-3173, USA

2 Oxford Internet Institute, University of Oxford, 1 St Giles, Oxford OX1 3JS, UK general discussion of philosophy of information as a new sub-discipline of philosophy (Adams and de Moraes), to philosophy of mind (Gamez), to philosophy of biology and medicine (Illari and Russo), to ethics and social philosophy (Rainey; Taddeo), to philosophy of action (Schulz), to information itself (Gobbo and Benini), and to semantics (Primiero and Kosolosky). What follows is a brief summary of each paper in this issue.

Fred Adams and João Antonio de Moraes critically evaluate the philosophy of information framework in their paper, Is There a Philosophy of Information? Their discussion examines the extent to which Philosophy of Information should be accepted as a new paradigm, and in what sense. They do this by comparing philosophy of information to other areas of philosophy, such as philosophy of biology or philosophy of mathematics. They also consider what the main issues are in philosophy of information and how they connect to other areas such as philosophy of mind and ethics. They conclude that philosophy of information not only provides a fresh look at traditional philosophical problems by using contemporary concepts from the theory of information, but that philosophy of information investigates genuinely new philosophical problems. Consequently, they argue that philosophy of information is an autonomous area of investigation in philosophy.

In their paper, Information channels and biomarkers of disease, Phyllis Illari and Federica Russo apply the informational approach to an issue concerning the nature of causality in the context of biomarkers of disease. Environmental factors are important causes of disease, but in order to carry out precise measurements we need to improve our understanding of the underlying causal links. In turn, this requires a clear philosophical understanding of the causal claims that are being made. One of the challenges of this work is to offer a concept of causal linking 
from macro to micro factors. Illari and Russo argue that the approach of Russo and Williamson, which emphasizes the need for evidence of difference-making and mechanisms, is too coarse-grained to offer such a concept. Illari and Russo build on previous work by Illari and argue instead for the concept of productive causality. In brief, causal production is seen as information transmission, similar to Salmon's mark transmission account, but without its weaknesses. While the account of causal production that Illari and Russo provide is thin, it is sufficiently robust to help with the conceptual needs presented in the science of biomarkers of disease. More specifically, the need is to conceptualize the linking of non-homogenous factors, e.g., how environmental ('macro') factors affect molecular ('micro') factors. This issue cannot be addressed simply by changing scales of measurement; it requires continuous linking between different types of causes and effects at different levels of scale. Illari and Russo argue that an information transmission approach to productive causality meets this need, and that this approach illustrates the first steps towards a full-blown informational approach to productive causality.

Stephen Rainey extends the program of the philosophy of information to a socio-political context in his paper The Method of Levels of Abstraction in Pluralism and Governance in Dialogical Interaction. His target is the governance of contentious issues, particularly in the context of European governance. In this context there is a pluralism of perspectives on various issues that is recognized. More specifically, European governance deploys a suitable level of formalism in the hope that differences of opinion, perspective, viewpoint, value, etc. will become practically irrelevant and will be overridden by solidarities of interests. This approach assumes that the content of many interactions eventually work themselves out, given that the members of the interactions are rational individuals a la Mill's homo oeconomicus. Rainey uses levels of abstraction, a central concept in philosophy of information, to better understand this approach. A level of abstraction (LoA) is a set of observables deemed relevant with respect to some system. In this case, the observables are the purposes, interests, and conceptual schemes of the people in dialogue. One of the advantages of this approach is that it meets the challenge that a dividual self presents to the formalist approach. The formalist approach assumes a classical individual, an enlightenment view where the self is the ultimate bearer of fundamental rational views. The approach of the dividual self, or better called an inforg in the light of the information revolution, lets the idea of pluralism be internal as well as external. Rainey illustrates this approach with two examples, one concerning freedom, the other concerning tax.

In their paper What can we know of computational information? Measuring, quantity, and quality at work on programmable artifacts Federico Gobbo and Marco Benini apply the concept of levels of abstraction (LoA) to the analysis of computational information. In particular, they focus on the conceptual issues that arise when using the concepts of quantity and quality to measure computational information. For example, measuring computational complexity is not merely a matter of quantification as classically understood, but is more nuanced. Also, the classical notion of quality is unsatisfying in a world where information and communication technologies are pervasive. Gobbo and Benini draw from the view of Informational Structural Realism to better understand the act of measuring.

Mariarosaria Taddeo's paper Just Information Warfare deals with ethical issues present in information warfare, warfare waged in the cyber domain. Taddeo argues that Just War Theory is a necessary instrument for doing an ethical analysis of information warfare, but it is not sufficient. Just War Theory draws on tenets such as last resort or more harm than good to determine when warfare is justified. Such tenets focus on the kinetic aspects of violent warfare, such as physical damage and bloodshed. Information warfare, however, includes a non-physical ontology, thereby rendering different ethical problems. For this reason, Taddeo argues that a suitable analysis requires that Just War Theory be merged with Information Ethics. The domain of Information Ethics, the Infosphere is the whole realm of reality that falls under an informational perspective, including non-physical entities. This informational perspective is non-anthropocentric. It considers the blooming of the Infosphere to be the ultimate good, and its entropy (destruction or corruption) as the ultimate evil. Consequently, the duty of any moral agent in the Infosphere is determined by their contribution to the informational environment. Taddeo argues that the injection of Information Ethics into Just War Theory produces the "necessary granularity" to handle the issues posed by information warfare.

In Are Information or Data Patterns Correlated with Consciousness? David Gamez applies the method of levels of abstraction to research on the relationship between consciousness and the physical world. Scientists have focused on the neural correlates of consciousness by looking for correlations between first-person reports and data sets of physical measurements of the brain. A more recent proposal is to look for links between information states in the brain and consciousness. Gamez makes this proposal more precise by drawing on the distinctions between dedomena, data and information. He applies the method of levels of abstraction to describe how data can be measured at different levels of abstraction. Gamez is careful, however, to address the limitations of the proposal to correlate data patterns and consciousness and outlines foreseeable problems with the approach. For example, to 
show that a data pattern is correlated with consciousness, three conditions have to be met. First, the measurement does not depend on a subjective choice of a level of abstraction. Second, a data pattern is only present when consciousness is present, and is absent at all levels of abstraction for the unconscious brain. Gamez suggests that it is the latter part of this second condition that will prove to be challenging. And third, the data pattern is correlated with consciousness independently of the physical substrate in which it is instantiated. Given these conditions, Gamez is rather sceptical that the approach to correlate data patterns with consciousness will be success. He suggests an alternative: to focus on correlations between consciousness and spatiotemporal patterns at particular levels of abstraction.

In An Informational Perspective on Agency Causation Christoph Schulz uses Fred Dretske's analysis of becoming informed to handle objections against the theory of agency. Such objections, as given by Peter Menzies and Huw Price, are concerned with the conceptual circularity and conceptual regress in the application of the concept of causation in the context of agency. In particular, the concern is that the notion of "bringing about" is itself a causal notion. Schulz offers an alternative interpretation that avoids this objection by working through an account of becoming informed. Having avoided the problem of conceptual circularity,
Schulz then expands his analysis to a wider range of problems that involve the relation between information and causation.

Finally, Giuseppe Primiero and Laszlo Kosolosky offer a formal treatment of the semantics of both complete and incomplete mistrustful or distrustful information transmission in their paper The Semantics of Untrustworthiness. The concept of trust is subject to various interpretations, depending on one's psychological, social, epistemic, or formal perspective. Primiero and Kosolosky focus on negative assessments of trust. They adopt a formal perspective to clarify the difference between distrust and mistrust. More specifically, they characterize trust as a second-order property of relations. By doing so, they can provide a conceptual analysis that is sufficiently finegrained between distrust and mistrust, which cannot be done if trust is characterized as a first-order relation. Their characterizations of distrust and mistrust provide the basis for understanding untrustworthy transmissions of both complete and incomplete information.

This collection of essays shows the scope and depth of new research in the philosophy of information, an area where theoretical and applicable philosophy meet. It has been a pleasure and privilege to work with so many colleagues and we hope the readers will find this special issue as interesting as we did. 